\title{
Fast inference of couplings between integrate-and-fire neurons from their spiking activity Simona Cocco ${ }^{1,2}$, Stanislas Leibler 3,2 and Rémi Monasson*3,2
}

\author{
Address: ${ }^{1}$ CNRS-Laboratoire Physique Statistique de l'ENS, Paris, 75005, France, ${ }^{2}$ The Rockefeller University, New York, USA and ${ }^{3}$ CNRS- \\ Laboratoire Physique Theorique de l'ENS, Paris, 75005, France \\ Email: Rémi Monasson* - monasson@lpt.ens.fr \\ * Corresponding author
}

from Eighteenth Annual Computational Neuroscience Meeting: CNS*2009

Berlin, Germany. 18-23 July 2009

Published: 13 July 2009

BMC Neuroscience 2009, I0(SuppI I):PI28 doi:I0.I |86/I47|-2202-I0-SI-PI28

This abstract is available from: http://www.biomedcentral.com//47I-2202/I0/SI/PI28

(C) 2009 Cocco et al; licensee BioMed Central Ltd.

Multi-electrode recordings make available the simultaneous spiking activity of tens of neurons for hours [1]. An important issue is to reconstruct the network of connections between the cells from this activity. To be more precise, let us model cells as Leaky Integrate-and-Fire (LIF) neurons (see [2] and references therein) whose membrane potentials obey the differential equation (units are chosen so that the membrane capacitance equals unity),

$$
\frac{d V_{i}}{d t}(t)=-g V_{i}(t)+\sum_{j} J_{i j} \sum_{k} \delta\left(t-t_{j k}\right)+I_{i}+\eta_{i}(t)
$$

where $g$ is the leak conductance. $J_{i j}$ is the strength of the connection from neuron $j$ onto neuron $i$ and $t_{j, k}$ the time at which cell $j$ fires its $k^{\text {th }}$ spike; we assume that synaptic inputs are instantaneously integrated i.e. the synaptic integration time is much smaller than $1 / g$ and the typical inter-spike interval. $I_{i}$ is a constant external current flowing into cell $i$, and $\eta_{i}(\mathrm{t})$ is a fluctuating current, modeled as a Gaussian white noise process with variance $\sigma^{2}$. Neuron $i$ remains silent as long as $V_{i}$ remains below some threshold potential (equal to1). If the threshold is reached at some time then a spike is emitted, and the potential is reset to its rest value of zero, and the dynamics resumes.

The above model implicitly defines the likelihood $P$ of the spiking times $\left\{t_{j, k}\right\}$ given the currents $I_{i}$ and synaptic couplings $J_{i j}$. If we are given the spike times $\left\{t_{j, k}\right\}$ we will infer the couplings and currents by maximizing $P$. In principle, $P$ can be calculated through the resolution of FokkerPlanck equations (one for each inter-spike interval) for a one-dimensional Orstein-Uhlenbeck process with moving boundaries. However, this approach, or related numerical approximations [3], are inadequate to treat data sets with hundreds of thousands of spikes (such as in recordings of populations of retinal ganglion cells) in a reasonable time.

In our approach, we choose to approximate $P$ from the contribution coming from the most probable trajectory for the potential for each cell $i$, referred to as $V_{i}{ }^{*}(t)$. This approximation is exact when the amplitude $\sigma$ of the noise is small. The determination of $V_{i}^{*}(t)$ was done numerically by Paninski for one cell in [4]. We have found a fast algorithm to determine $V_{i}{ }^{*}(t)$ analytically in a time growing linearly with the number of spikes and quadratically with the number of neurons, which allows us to process recordings with tens of neurons easily. The algorithm is based on a detailed and analytical resolution of the coupled equations for the optimal potential $V_{i}{ }^{*}(t)$ and the associated optimal noise $\eta_{i}{ }^{*}(t)$ through (1), and is too complex to be explained in this abstract.

Once the optimal paths for the potential and noise has been determined, we calculate the log-likelihood of the corresponding couplings and currents through the integral of the squared optimal noise [4]. This log-likelihood is clearly a concave function of the currents and couplings 
and can be easily maximized using the Newton-Raphson method. Our algorithm has been tested on artificially generated data, real experimental data (recordings of 32 ganglion cells in the salamander retina submiited to random flickering stimulus, courtesy of M. Meister) and compared to other inference methods based on the Ising model (see abstract by Cocco). To give a flavor of the computational effort required, it takes us about 20 seconds to process a set of 120,000 spikes fired by 32 cells on a personal computer.

\section{References}

I. Taketani M, Baudry L: Advances in Network Electrophysiology Using MultiElectrode Arrays Springer-Verlag, New York; 2006.

2. Jolivet R, Lewis TJ, Gertsner W: Generalized integrate-and-fire models of neuronal activity approximate spike trains of a detailed model to a high degree of accuracy. J Neurophys 2004, 92:954.

3. Paninski L, Pillow JW, Simoncelli EP: Maximum likelihood of a stochastic integrate-and-fire neural econding model. Neural Computation 2004, 16:25-53.

4. Paninski L: The most likely voltage path and large-deviation approximations for integrate-and-fire neurons. J Comput Neurosci 2006, $21: 71$.
Publish with Bio Med Central and every scientist can read your work free of charge

"BioMed Central will be the most significant development for disseminating the results of biomedical research in our lifetime. "

Sir Paul Nurse, Cancer Research UK

Your research papers will be:

- available free of charge to the entire biomedical community

- peer reviewed and published immediately upon acceptance

- cited in PubMed and archived on PubMed Central

- yours - you keep the copyright

Submit your manuscript here:

http://www.biomedcentral.com/info/publishing_adv.asp 